\title{
La orientación intercultural: Una perspectiva para favorecer la convivencia en contextos multiculturales
}

\author{
Intercultural Counseling: A Perspective to Favor Coexistence in Multicultural Contexts
}

\author{
Ana Victoria Garita Pulido ${ }^{1}$ \\ Colegio Técnico Profesional de Pavas \\ San José, Costa Rica \\ avictoria@gmail.com
}

Recibido 29 de agosto de 2013 • Corregido 27 de octubre de 2013 • Aceptado 6 de noviembre de 2013

\begin{abstract}
Resumen. Este artículo hace alusión al ejercicio profesional de la orientación con perspectiva intercultural para el abordaje de contextos multiculturales. Reflexiona sobre la convivencia como un desafío social en contextos multiculturales, analiza la trascendencia de la cultura en la conformación de la identidad personal y, por ende, la necesidad de que sea considerada en toda acción orientadora. Presenta los principios, propósitos y características de la perspectiva intercultural como modelo integrador para la atención de la diversidad cultural, junto con algunos argumentos para delimitar la orientación intercultural, asimismo las actitudes, conocimientos y habilidades que la persona orientadora ha de desarrollar para ser interculturalmente competente. Además, plantea aquellas competencias que se han de promover en las poblaciones que atienden, para crear una interacción de convivencia positiva a partir de las diferencias.
\end{abstract}

Palabras claves. Convivencia, identidad, diversidad cultural, orientación, interculturalidad.

Abstract. This article refers to the professional practice of Counseling with an intercultural perspective to face multicultural contexts. The paper reflects on coexistence as a social challenge in multicultural contexts and analyzes the importance of culture in the creation of personal identity, and the resulting need for it to be considered in every counseling action. Principles, purposes and characteristics of the intercultural perspective are presented as an integrative model for the attention of cultural diversity, as well as some arguments to define intercultural counseling. The attitude, knowledge and skills to be developed by counselors to be interculturally competent are also mentioned in the paper. In addition, the study lists all those skills that should be promoted in the counselor's clients to create a positive coexisting interaction based on their differences.

Keywords. Coexistence, identity, cultural diversity, counseling, interculturality.

1 Magistra en Orientación de la Universidad de Costa Rica. Bachiller y licenciada en Ciencias de la Educación con énfasis en Orientación de la Universidad Católica de Costa Rica. Ha laborado como orientadora en centros de educación secundaria y como docente de la carrera de Orientación Educativa de la Universidad Católica de Costa Rica. Actualmente es miembro del Tribunal de Honor del Colegio de Profesionales en Orientación y docente de la Maestría en Orientación con énfasis en Orientación Laboral de la Universidad de Costa Rica. 
URL: http://www.una.ac.cr/educare

CORREO: educare@una.cr

\section{Aprender a convivir: Un desafío en contextos multiculturales}

La compleja realidad social vinculada a las altas tasas de desplazamiento de personas pertenecientes a diferentes culturas y al reconocimiento de la multiculturalidad existente en un territorio particular pone de manifiesto que la convivencia es un nuevo desafío social.

Este desafío surge de la consideración de que "las sociedades son cada vez más diversas, ya que en la actualidad conviven gentes de distintas razas y diferentes culturas. Esta situación se ve incrementada de forma progresiva debido a las tendencias migratorias que se van produciendo en el mundo" (Gallardo, 2009, p. 125).

Esta consideración visibiliza la naturaleza multicultural existente en todo contexto social, hecho que supone acciones conducentes a la creación de contextos sociales caracterizados por una convivencia cada vez más armoniosa, incluyente, participativa y equitativa.

Dichas acciones se enfocan en que las personas aprendan a convivir, sustentadas en "el desarrollo de la comprensión de los otros en un espíritu de tolerancia, pluralismo, respeto de las diferencias y la paz (...) de la interdependencia creciente (ecológica, económica, social) de las personas, las comunidades y las naciones en un mundo cada vez más pequeño, frágil e interconectado" (Organización de las Naciones Unidas para la Educación, la Ciencia y la Cultura [UNESCO], 2003, p. 29).

Una interacción social positiva caracterizada por el respeto, la tolerancia y la interdependencia debe partir del reconocimiento de las diferencias, de la inclusión y participación social de todas las personas en un contexto particular.

La convivencia entendida como un desafío en una sociedad multicultural conlleva a que desde las diversas instituciones y actores sociales se implementen acciones tendientes a favorecer la creación de espacios de interacción social positiva, basados en el reconocimiento y valoración real de la diferencia, de manera que se favorezcan espacios en que se fomente la comprensión mutua, basada en principios de respeto a la diversidad, la solidaridad e interdependencia entre diferentes personas, grupos culturales y naciones (UNESCO, 2003).

Para la consecución de dicho propósito, y como una forma de ampliar su quehacer, la orientación como disciplina y profesión tiene una responsabilidad social, que en este caso consiste en implementar acciones para que las personas aprendan a convivir en los contextos en que se desarrollan. Esta tarea supone ampliar la práctica profesional con referentes teóricos más integradores que permitan la comprensión de las diferencias culturales presentes en dichos contextos, de manera que esta se ajuste a las características y necesidades particulares de las poblaciones que se atienden. 


\section{Las culturas como foco de atención en la intervención orientadora}

La cultura es un elemento determinante en la conformación de la identidad personal, pues las características propias del grupo de pertenencia repercuten de forma directa en su conformación. Este aspecto resulta trascendente en cualquier acción orientadora, pues "la identidad es primero y sobre todo una pertenencia objetiva: al ser parte de un grupo la persona adquiere el carácter peculiar de ese grupo" (Martín-Baró, 1992, pp. 117).

Para conocer a las personas y las sociedades, se requiere reconocer y aceptar la diversidad cultural implícita; según el grupo cultural al que se pertenece, se aprende una cosmovisión e idiosincrasia particular, las cuales son reproducidas en los diversos contextos en que se interactúa.

De ahí que la cultura interviene en el desarrollo y bienestar personal y colectivo ya que "... produce en los seres humanos un modo de concebir el mundo, de pensar, percibir, hablar, sentir y valorarse a [sí] mismo, expresarse y comportarse, comunicarse y organizarse socialmente (Us Soc, 2002, p. 32).

Favorecer el bienestar personal y colectivo ha de ser el propósito de toda política pública $y$, por ende, de las diferentes instituciones y actores sociales que ejercen su labor en un contexto particular. Para ello, se requieren políticas que se traduzcan en acciones que visibilicen, respeten y favorezcan a los diversos grupos culturales y étnicos que coexisten en un determinado territorio.

En el caso de la orientación -que busca coadyuvar en ese bienestar y se ejerce en contextos muy particulares-, entender y discernir la realidad cultural de las personas y su trascendencia para el desarrollo integral es un requerimiento fundamental para la intervención (Repetto, 2001). No obstante, en algunas ocasiones -ocurre en la prácticase atiende a la persona desenraizándola de su contexto, por tanto, de los elementos constitutivos de su identidad, los cuales vienen dados por su grupo cultural y lugar de pertenencia.

Esto demanda modelos teóricos con mayor influjo social que le permitan, a la persona orientadora, conocer los elementos de tipo social, político, cultural, económico y axiológico que permitan vislumbrar la diversidad de estilos de vida de las poblaciones que atiende (Gavilán, 2006), según el lugar y cultura de procedencia.

El reconocimiento de la realidad multicultural de los diversos contextos en que se ejerce la orientación pone de manifiesto la importancia de emprender acciones ajustadas a sus necesidades y características particulares. Esto implica que, para trabajar desde esta perspectiva, se hace necesario contar con un marco de referencia conforme a las diversas realidades socioculturales, para dar una respuesta efectiva a los retos que estas generan. 
URL: http://www.una.ac.cr/educare

CORREO: educare@una.cr

\section{La perspectiva intercultural: un referente para la acción orientadora}

Reconocer que un contexto social es culturalmente diverso supone que el abordaje por parte de los actores sociales no puede quedar sujeto a una visión simplista y estática de la realidad. Implica no solo reconocer la complejidad de ese contexto multicultural, sino también lo que demanda para fomentar acciones que fortalezcan la convivencia armónica entre las personas, a partir del significado y comprensión de las diferencias. Conlleva asumir que "la diversidad es la norma, que todo grupo humano es diverso culturalmente y puede ser descrito en función de sus características culturales ... de sus diferentes formas de estar, ver y construir el mundo, la realidad, sus relaciones con los demás ..." (Aguado, 2003, p. 14).

Esa conceptuación de la diversidad cultural conduce a considerar lo diverso como un aspecto inherente a la naturaleza humana, como fuente de crecimiento y enriquecimiento personal y colectivo, que delimita nuevos derroteros en las acciones que se emprenden para atenderla de manera adecuada.

Desde esta perspectiva, la interculturalidad se plantea como un modelo integral para atender la diversidad cultural que se concibe como:

[Una alternativa] socio política propositiva muy clara expresada en acciones tendientes al reconocimiento de las posibilidades y riquezas de nuestra diversidad, el sostenimiento de nuestras particularidades y la lucha frontal contra las desigualdades instaladas en la sociedad en pos de aportar a la solución de conflictos entre culturas y a la transformación del marco estructural que origina inequidad política, socio-económica y cultural (Vásquez, 2007, p. 1).

La interculturalidad conlleva una posición epistemológica que considera lo diverso como un aspecto positivo, como fuente de desarrollo personal y social. Como acción propositiva supone la realización de esfuerzos vinculados a un cambio ideológico en el sistema social, al pretender la construcción de sociedades más equitativas y participativas, en las que la convivencia sea en equidad e igualdad de oportunidades con principios de respeto, aceptación e inclusión.

Por ello, la perspectiva intercultural no puede ser reducida a la atención de las necesidades exclusivas de grupos culturales que sean objeto de exclusión social, sino más bien constituye:

La propuesta de una sociedad que abre sus ojos a la diversidad cultural interna y mundial y que hace de esta diferencia el tema central en su propuesta. Apunta a una transformación social que busca el tránsito de una dinámica social movida por la dominación y la negación de las diferencias culturales a una dinámica social que promueva afirmación identitaria y acercamientos, préstamos y fusiones entre culturas en equidad de condiciones. (Vásquez, 2007, p. 4) 
URL: http://www.una.ac.cr/educare CORREO: educare@una.cr

Esta perspectiva pretende romper con el esquema de desigualdad y exclusión imperante entre grupos culturales, apuesta al abordaje del total de la población considerando sus particularidades y fortaleciendo identidades, con el propósito de inducir a una convivencia social adecuada, en un marco de respeto y valoración positiva de las diferencias. De acuerdo con Giménez (2003) y Aguado (2003) algunas de sus características son:

- Sitúa en el foco de la reflexión la diversidad cultural, la considera un constructo dinámico, cambiante y adaptativo. Asume las diferencias como la norma, no como una deficiencia.

- Busca las convergencias sobre las cuales establecer vínculos y puntos en común entre las diversas culturas. Pone énfasis en el aprendizaje mutuo, la cooperación y el intercambio.

- Promueve la convivencia entre diferentes, trata de construir la unidad en la diversidad. Propicia el descubrimiento, encuentro, confrontación, el reconocimiento y aceptación de las otras personas.

- Se orienta al desarrollo de competencias en todos los ámbitos sociales, con el fin de superar la desigualdad, el racismo y la discriminación social.

Enfatiza en la interacción y en la valoración positiva de lo diferente, su fin es provocar en las personas un aprendizaje para la convivencia en equilibrio, lo cual lleva a la creación de contextos cada vez más armoniosos en los que esas personas puedan convivir en equidad e igualdad de condiciones.

El abordaje con perspectiva intercultural representa un accionar sistemático y unificado que constituye un proceso político y social que aglutina los esfuerzos del estado y de la sociedad civil para alcanzar tales propósitos. Supone:

La promoción sistémica y gradual, desde el Estado y desde la sociedad civil, de espacios y procesos de interacción positiva que vayan abriendo y generalizando relaciones de confianza, reconocimiento mutuo, comunicación efectiva, diálogo y debate, aprendizaje e intercambio, regulación pacífica del conflicto, cooperación y convivencia..., en el contexto de un Estado democrático, participativo y de una Nación multicultural, multilingüe y multiétnica. (Us Soc, 2002, p. 52)

La realidad multicultural de los contextos en que se ejerce la orientación exige incorporar, en el cuerpo de conocimientos que la sustentan, modelos integradores que permitan comprenderla para brindar una atención oportuna y pertinente a las poblaciones a quienes dirige su accionar, considerando la diversidad existente. 
URL: http://www.una.ac.cr/educare

CORREO: educare@una.cr

Implementar con éxito la perspectiva intercultural constituye, para la orientación como disciplina y profesión, una demanda de sentido, porque involucra trascender las instituciones, los espacios y áreas disciplinares, pues resulta tarea para todos los actores e instituciones sociales.

\section{La orientación intercultural: La necesidad de infundir la perspectiva intercultural en el ejercicio profesional}

Asumir la perspectiva intercultural como referente en el ejercicio profesional resulta una tarea impostergable para la persona orientadora, pues las diferencias culturales son una realidad que no se puede ignorar (Repetto, 2001). Dicha perspectiva le posibilita facilitar procesos vinculados con la convivencia adecuada a partir de las diferencias y provee a la persona orientadora de un marco referencial que reconoce las culturas y su trascendencia en el desarrollo personal y social.

Repetto (2001) hace referencia a lo que implica asumir la perspectiva intercultural en la labor orientadora:

a) Circunscribir los enfoques, teorías y metodologías a un contexto particular.

b) Reconocer que en la relación de ayuda dos o más de las personas participantes pertenecen a distintas culturas.

c) Contextualizar la intervención con los elementos que caracterizan las culturas presentes en la relación de ayuda.

d) Adecuar culturalmente los conocimientos, destrezas y actitudes en la ayuda profesional.

La perspectiva intercultural en los servicios de orientación resulta necesaria, pues implica "una perspectiva dinámica de la diversidad cultural... se centra en el contacto y la interacción, la influencia mutua" (Aguado, 2003, p. 14), propiciando contextos de sana convivencia.

Para la persona orientadora el punto de partida -como el de llegada-es contextualizar las acciones que emprende, en términos de que estas involucren las particularidades culturales de las personas a quienes dirige su accionar. Obviar este presupuesto es continuar invisibilizando la relación dialéctica persona -entorno, descontextualizando a la persona de su grupo cultural de pertenencia y, con ello, omitiendo en la intervención los atributos culturales, sociales, políticos, axiológicos, ambientales, entre otros, que conforman su identidad y su estilo de vida.

Contextualizar la acción orientadora implica un proceso de reflexión acerca de la trascendencia de la cultura en el desarrollo personal y colectivo. Das (2000), citado por Sanz (2001), propone algunas interrogantes para la reflexión conducentes a la contextualización de la práctica orientadora: 
URL: http://www.una.ac.cr/educare CORREO: educare@una.cr

- ¿Cómo la cultura condiciona la conducta de todos los seres humanos?

- ¿Cómo las normas, valores y expectativas culturales pueden crear dificultades para las cuales las personas buscan solución?

- ¿Cómo cada cultura desarrolla una serie de mecanismos para ayudar a los individuos a enfrentarse a sus problemas?

- ¿Cómo surgen dificultades en una sociedad multicultural? ¿Cómo se pueden atender esas dificultades?

Esta reflexión facilita que la diversidad cultural sea un eje transversal de las múltiples acciones que la persona orientadora desarrolle en un contexto determinado, pues la perspectiva intercultural no se reduce a la aplicación de programas de orientación, sino a infundirla en todas las dimensiones del ejercicio profesional (Repetto, 2001), es decir, incorporarla al desarrollo de una orientación intercultural.

Aunado a la reflexión sobre la valoración de la cultura y su impacto en la configuración de la identidad personal y colectiva, la persona orientadora requiere de un análisis previo o simultáneo enfocado en sí misma, por cuanto como persona también ha sido condicionada por el grupo cultural al que pertenece. Malik (2003) señala una serie de competencias interculturales por desarrollar para una adecuada atención a la diversidad cultural:

- Actitudes interculturales: referidas a la curiosidad, apertura, aceptación, disposición, voluntad para cuestionar los propios valores, creencias y comportamientos asumiendo que no son los únicos o correctos.

- Conocimientos: de los grupos sociales, de sus costumbres, el conocimiento de otras personas, de cómo se ven a sí mismas, de los procesos de interacción social, de cómo funcionan y cómo se forman los grupos sociales y sus identidades.

- Habilidades o destrezas: relacionadas con la interpretación y comparación de perspectivas, hechos, ideas o documentos de otras culturas y relacionarlos con la propia cultura. A la vez con el aprendizaje e interacción que permitan aprender de las diversas culturas y favorecer la interacción.

Como puede verse, ser interculturalmente competente implica para la persona orientadora atender, desde un marco de respeto y valoración positiva, la diversidad cultural existente en los contextos en que labora, asumir una actitud de apertura ante las múltiples identidades que confluyen en estos $y$, a la vez, tomar conciencia de sí misma reconociendo su propio bagaje familiar, social y cultural, y ocuparse de que este no interfiera en la práctica profesional. 
URL: http://www.una.ac.cr/educare

CORREO: educare@una.cr

Esto permite a la persona orientadora alcanzar un nivel adecuado de competencia intercultural, traducido en el desarrollo de acciones que favorezcan, en las poblaciones meta, habilidades y destrezas para interactuar positivamente en ambientes culturalmente diversos. La suma de dichas acciones converge hacia la consecución de competencias interculturales por parte de las personas destinatarias, que de acuerdo con Malik (2003) son:

- Eficacia intercultural: vinculada con la comprensión y conocimiento de la propia cultura, la conciencia de la existencia y validez de otras culturas, las habilidades para mantener relaciones interculturales eficaces, la identificación de pautas comunicativas y de actitudes discriminatorias, el conocimiento de las características y códigos de otras culturas.

- Comprensión de las diferenciasyrespeto hacialadiversidad:relacionado con la consciencia y comprensión de la diversidad que caracteriza a personas y grupos sociales, el análisis de los valores personales respecto al sexismo, el racismo, prejuicio y discriminación, el reconocimiento y cuestionamiento de los estereotipos y la comprensión de su impacto en la propia conducta y en la de otras personas.

Estas competencias facilitan, en las personas destinatarias, posibilidades para interactuar de manera adecuada con otras personas y en contextos diversos en un marco de tolerancia, respeto y aceptación, lo que constituye un insumo para la construcción de identidades y sociedades cada vez más inclusivas, equitativas, democráticas y participativas, desde el supuesto básico de la valoración positiva de la diferencia.

La perspectiva intercultural delimita nuevos derroteros para la orientación como disciplina y profesión que representan, como ya se mencionó, una demanda de sentido vinculada a la ampliación de sus referentes teóricos y metodológicos. Para Rojas (2007, p. 250) esta ampliación se relaciona con "un mirar de la Orientación y su acción desde la complejidad humana ... teniendo así una visión de ser humano físico, biológico, psíquico, antropológico, revestido de una personalidad, en constante interacción con lo cultural y lo social".

De manera que se pueda transitar de una visión psicológica de la persona, hacia una que además aporte elementos biológicos, sociológicos-antropológicos y culturales para la comprensión de la complejidad humana y social; que posibilite el ejercicio de una orientación realmente intercultural.

Esto implica la revisión y reestructuración de la propuesta curricular de la carrera de Orientación impartida por las instituciones de educación superior, de manera que se incluya como parte de la formación, temas con mayor influjo social relacionados con los procesos de identidad y socialización, la noción de cultura, el conocimiento de las culturas y de los procesos sociales, la perspectiva intercultural, la formación en competencias interculturales, entre otros. 
URL: http://www.una.ac.cr/educare CORREO: educare@una.cr

Para las personas profesionales que ya ejercen la orientación, supone actualizarse en dichas temáticas de manera que se les facilite una visión centrada en la complejidad de las personas y de los contextos en los cuales se desempeñan.

\section{Consideraciones finales}

La naturaleza multicultural de todo contexto social representa una demanda de sentido para todas las instituciones y actores sociales vinculados con la convivencia social positiva. Así la persona orientadora debe incorporar a su quehacer acciones que promuevan el reconocimiento, respeto y valoración positiva de las diferencias.

El grupo cultural de pertenencia influye en la conformación de la identidad mediante procesos de socialización desde los cuales se modela a las personas que lo integran, en costumbres, valores, tradiciones; en la forma de percibirse a sí mismas, de ver el mundo, de relacionarse, de expresarse y de convivir.

Las culturas cobran relevancia para la orientación como disciplina y profesión por su trascendencia en el desarrollo personal y colectivo. Realidad que la persona orientadora no puede obviar, si desea que sus acciones sean oportunas y pertinentes a las necesidades y características particulares de las personas en general y de las que atiende en particular.

La perspectiva intercultural pretende la convivencia armónica a partir de la diferencia; surge como una propuesta para la atención a la diversidad cultural, basada en principios de inclusión, respeto, tolerancia, equidad y valoración positiva de la diferencia.

Esto denota un posicionamiento epistemológico frente a la diferencia, el cual se vinculada a su consideración como un aspecto real y positivo inherente a la naturaleza humana. Conlleva acciones que pretenden reducir las desigualdades y la exclusión, fortalecer identidades y crear entornos sociales de sana convivencia. Representa una propuesta que debe ser llevada a cabo desde el Estado y la sociedad civil, por lo que la orientación tiene una responsabilidad social que cumplir ante la realidad multicultural de los contextos en que se circunscribe su accionar.

Asumir la perspectiva intercultural supone, para la persona orientadora, impregnar toda su intervención con sus fundamentos; involucra una inclusión de la interculturalidad en los enfoques; las teorías; la conceptuación de la relación de ayuda y de las personas destinatarias, los conocimientos, habilidades y destrezas; todo lo cual conlleva a la contextualización de la práctica profesional.

A la vez, incluye tomar conciencia de los valores, actitudes, prejuicios, comportamientos, entre otros, propios del acervo cultural de la persona orientadora, pues podrían interferir en su práctica profesional. Esto conduce a un ejercicio profesional interculturalmente competente, que implica para la persona orientadora ser consciente de su propio bagaje sociocultural, tener apertura a la diversidad cultural existente en un contexto particular y contextualizar su práctica culturalmente. 
URL: http://www.una.ac.cr/educare

CORREO: educare@una.cr

Estas acciones confluyen en la adquisición de competencias interculturales por parte de las personas destinatarias de la acción orientadora, vinculadas con la eficacia para interactuar en contextos multiculturales y a la comprensión de las diferencias y el respeto a la diversidad.

Para la formación profesional representa la inclusión de temas con mayor influjo social que faciliten una visión dinámica de la persona como un todo indivisible en continua interacción con su entorno, a partir de referentes sociológicos-antropológicos. Para las personas que ya ejercen la orientación, la formación continua les debe permitir una visión de la complejidad humana y de los contextos sociales donde llevan a cabo su labor.

Los aspectos sobre los que se ha reflexionado en este artículo constituyen solo una propuesta producto del interés personal y profesional, surgida de la realidad social compleja y de la experiencia laboral en contextos donde interactúan personas de diversas culturas. Como tales, pretenden abrir la discusión referente a las implicaciones de la diversidad cultural de los contextos en que se ejerce la orientación y el desarrollo de una orientación intercultural.

\section{Referencias}

Aguado, T. (2003). Pedagogía intercultural. Madrid: McGraw-Hill.

Gallardo, P. (marzo, 2009). Educación ciudadana y convivencia democrática. Revista Interuniversitaria de Pedagogía Social, 16. Recuperado de http://www.upo.es/revistas/ index.php/pedagogia social/article/view/39/35

Gavilán, M. (2006). La transformación de la orientación vocacional: Hacia un nuevo paradigma. Argentina: Homo Sapiens Ediciones.

Giménez, C. (2003). Pluralismo, multiculturalismo e interculturalidad: Propuesta de clarificación y apuntes educativos. Educación y Futuro. Revista de Investigación Aplicada y Experiencias Educativas, 8, 1-24. Recuperado de http://dialnet.unirioja.es/servlet/ ejemplar?codigo $=138249$

Malik, B. (2003). Intervenciones para la adquisición de competencias interculturales. En E. Repetto (Direct.), Modelos de orientación e intervención psicopedagógica (Vol. II Intervenciones psicopedagógicas para el desarrollo del aprendizaje, de la carrera de la persona, pp. 424452). Madrid: UNED.

Martín-Baró, I. (1992). Acción e ideología: Psicología social desde Centroamérica. El Salvador: UCA Editores. 
Organización de las Naciones Unidas para la Educación, la Ciencia y la Cultura [UNESCO] (Setiembre, 2003). Aprender a vivir juntos: ¿hemos fracasado? Síntesis de las reflexiones y los aportes surgidos durante la $46^{\mathrm{a}}$ Conferencia Internacional de Educación de La UNESCO Ginebra: Autor. Recuperado de http://www.ibe.unesco.org/fileadmin/user upload/archive/publications/free publications/ICE 2001 summary sp.pdf

Repetto, E. (2001). La orientación intercultural: Problemas y perspectivas. Recuperado de http:// www.aulaintercultural.org/IMG/pdf/elvira.pdf

Rojas, L. (enero-junio, 2007). Reflexiones para una nueva visión de la orientación. Revista Ciencias de la Educación, 1(29), 243-252. Recuperado de http://servicio.bc.uc.edu.ve/ educacion/revista/vol1n29/art15.pdf

Sanz, R. (2001). Orientación psicopedagógica y calidad educativa. Madrid: Pirámide.

Us Soc, P. (2002). La práctica de la interculturalidad en el aula. San José, Costa Rica: CECC/ SICA. Recuperado de http://www.ceducar.info/CEDUCAR/recursos/biblioteca\%20online/ Volumen\%2013/HTML/index.html

Vásquez, J. A. (2007). Enfoque intercultural para una educación básica regular intercultural y bilingüe. Revista Cultural Electrónica Construyendo Nuestra interculturalidad, 4, 1-13. Recuperado de http://interculturalidad.org/numero04/docs/0202-Enfoque InterculturalVasquez,Jose.pdf

\section{Cómo citar este artículo en APA:}

Garita, A. V. (enero-abril, 2014). La orientación intercultural: Una perspectiva para favorecer la convivencia en contextos multiculturales. Revista Electrónica Educare, 18(1), 281-291. Recuperado de http://www.revistas.una.ac.cr/index.php/EDUCARE/issue/current

Nota: Para citar este artículo en otros sistemas puede consultar el hipervínculo "Como citar el artículo" en la barra derecha de nuestro sitio web:

http://www.revistas.una.ac.cr/index.php/EDUCARE/index 